ORIGINAL ARTICLE

\title{
PERFORMANCE OF CONVENTIONAL PCRs BASED ON PRIMERS DIRECTED TO NUCLEAR AND MITOCHONDRIAL GENES FOR THE DETECTION AND IDENTIFICATION OF Leishmania spp.
}

\author{
Estela Gallucci LOPES(1), Carlos Alberto GERALDO JUNIOR(1), Arlei MARCILI(1), Ricardo Duarte SILVA(1), \\ Lara Borges KEID(2), Trícia Maria Ferreira da Silva OLIVEIRA(2) \& Rodrigo Martins SOARES(1)
}

\begin{abstract}
SUMMARY
In visceral leishmaniasis, the detection of the agent is of paramount importance to identify reservoirs of infection. Here, we evaluated the diagnostic attributes of PCRs based on primers directed to cytochrome-B ( cytB), cytochrome-oxidase-subunit II (coxII), cytochrome-C $(c y t C)$, and the minicircle-kDNA. Although PCRs directed to $c y t B$, coxII, $c y t C$ were able to detect different species of Leishmania, and the nucleotide sequence of their amplicons allowed the unequivocal differentiation of species, the analytical and diagnostic sensitivity of these PCRs were much lower than the analytical and diagnostic sensitivity of the kDNA-PCR. Among the 73 seropositive animals, the asymptomatic dogs had spleen and bone marrow samples collected and tested; only two animals were positive by PCRs based on $c y t B$, coxII, and $c y t C$, whereas 18 were positive by the kDNA-PCR. Considering the kDNA-PCR results, six dogs had positive spleen and bone marrow samples, eight dogs had positive bone marrow results but negative results in spleen samples and, in four dogs, the reverse situation occurred. We concluded that PCRs based on $c y t B$, coxII, and $c y t C$ can be useful tools to identify Leishmania species when used in combination with automated sequencing. The discordance between the results of the kDNA-PCR in bone marrow and spleen samples may indicate that conventional PCR lacks sensitivity for the detection of infected dogs. Thus, primers based on the kDNA should be preferred for the screening of infected dogs.
\end{abstract}

KEYWORDS: Leishmania spp.; kDNA; Mitochondrial genes; PCR; Phylogeny; Dogs.

\section{INTRODUCTION}

The protozoan parasite that causes leishmaniasis belongs to the order Kinetoplastida, family Trypanosomatida and genus Leishmania ${ }^{1}$. The protozoan infects the mononuclear phagocyte system mainly in spleen, liver, bone marrow, and lymph nodes ${ }^{2}$.

There are reports of at least 30 different Leishmania species distributed throughout the Old and New World ${ }^{1,3}$. The species that cause leishmaniasis in Latin America are divided into two taxonomic groups. One group is the subgenus Viannia, which comprises the species Leishmania (Viannia) braziliensis, Leishmania (Viannia) panamensis and Leishmania (Viannia) guyanensis, and are responsible for cutaneous or mucocutaneous infections. The other group is the subgenus Leishmania, which includes the species Leishmania (Leishmania) mexicana and Leishmania (Leishmania) amazonensis, responsible for localized or diffuse skin lesions, and Leishmania (Leishmania) infantum, which causes visceral leishmaniasis ${ }^{1,4,5}$.

In Brazil, the most frequently encountered species is Leishmania
(Leishmania) infantum chagasi and the most commonly found vectors are Lutzomyia longipalpis and Lutzomyia cruzi $^{6,7}$. Based on molecular criteria, Mauricio et al. (1999) ${ }^{8}$ considered the species Leishmania (Leishmania) infantum chagasi and Leishmania (Leishmania) infantum synonymous. In the Americas, eleven dermotropic species of Leishmania causing disease in humans, and eight species described only in animals are currently recognized. In Brazil, seven dermotropic species have been identified, six of the subgenus Viannia and one of the subgenus Leishmania. The three main dermotropic species are: Leishmania (Viannia) braziliensis, Leishmania (Viannia) guyanensis and Leishmania (Leishmania) amazonensis. Recently, the species Leishmania (Viannia) lainsoni, Leishmania (Viannia) naiffi, Leishmania (Viannia) lindenberg, and Leishmania (Viannia) shawi were identified in North and Northeast States in Brazil ${ }^{9}$.

The Polymerase Chain Reaction (PCR) is largely used for the detection and identification of pathogens and is a valuable tool for molecular and epidemiological studies. In leishmaniasis, as in many other systemic infections, the detection of the agent is of paramount importance for the confirmation of infection in seropositive animals, and in studies carried 


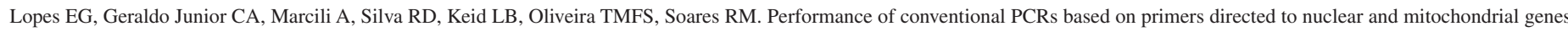
for the detection and identification of Leishmania spp. Rev Inst Med Trop Sao Paulo. 2016;58:41.

out with vectors and free-living animals to identify reservoirs of infection. In addition, PCR allows the molecular identification of the agent when phylogenetically-informative markers are amplified and sequenced, such as DNA barcode within mitochondrial and nuclear genomes ${ }^{10,11}$. Mitochondrial genes have shown a high degree of resolution in molecular/ phylogenetic studies because they are a result of maternal transmission in metazoans ${ }^{12}$. Besides, mitochondrial genes phylogenies are not affected by multiple nucleotide substitutions driven by adaptive selection, since the majority of the substitutions found in these loci are synonymous ${ }^{13}$.

The aim of the present study was to determine the analytical and diagnostic performance of PCRs based on primers directed to conserved markers located in the mitochondrial (-maxicircle-kDNA and minicircle$\mathrm{kDNA}$ ) and the nuclear genome for the direct diagnosis of Leishmaniasis using animal tissues.

In addition, we aimed to compare the performance of a PCR based on the minicircle-kDNA detection in bone marrow and spleen samples for the post-mortem diagnosis of canine leishmaniasis (CVL) in seropositive, asymptomatic dogs. In this kind of animals (seropositive but asymptomatic), Leishmania testing is still controversial.

\section{MATERIAL AND METHODS}

\section{Parasites}

Promastigotes of Leishmania spp. grown in Schneider medium with $20 \%$ fetal bovine serum were used. The samples were provided by the Leishmaniasis Research Laboratory, Oswaldo Cruz. Foundation and belonged to the Collection of Leishmania of the Instituto Oswaldo Cruz (CLIOC).

The following reference strains were used: Leishmania (leishmania) mexicana (MHOM/BZ/1982/BEL21), Leishmania (Viannia) guyanensis (MHOM/BR/1975/M4147), Leishmania (Viannia) braziliensis (MHOM/ BR/1975/M2903), Leishmania (Leishmania) tropica (MHOM/SU/1958/ STRAIN OD), Leishmania (Leishmania) amazonensis (IFLA/BR/1967/ PH8), Leishmania (Viannia) lainsoni (MHOM/BR/1981/M6426), Leishmania (Viannia) naiffi (MDAS/BR/1979/M5533), Leishmania (Viannia) shawi (MCEB/BR/1984/M8408), Leishmania (Leishmania) donovani (MHOM/ET/1967/L82;HV3;LV9), Leishmania (Viannia) major (MHOM/IL/1980/FRIEDLIN), Leishmania (Leishmania) infantum chagasi (MHOM/BR/2002/LPC-RPV), Leishmania (Leishmania) infantum (MHOM/TN/1993/LV10), Leishmania (Leishmania) hertigi (MCOE/PA/1965/C8), Leishmania (Leishmania) infantum chagasi (MHOMBR/1974/PP75), and Leishmania (Viannia) colombiensis (IGOM/PA/1985/E582.34).

\section{Dog samples}

Samples of 73 CVL seropositive and asymptomatic dogs from the city of Espírito Santo do Pinhal, São Paulo State, were collected in 2010. The dogs were neither evaluated by radiological and ultrasound methods, nor by pathological ones. The asymptomatic condition was defined after visual inspection and the confirmation of the absence of emaciation, skin lesions, onychogryphosis, alopecia and hair loss.

The serological status of the animals was determined at the time of sampling using ELISA (EIE-leishmaniose-visceral-canina-BioManguinhos, Rio de Janeiro, RJ, Brazil) and IFAT (IFI-leishmaniosevisceral-canina-Bio-Manguinhos, Rio de Janeiro, RJ, Brazil) consecutively, as recommended by the Brazilian sanitary authorities. After euthanasia, the animals were necropsied and fragments of the spleen and aspirates of bone marrow were collected. The samples were kept in $1.5 \mathrm{~mL}$ plastic microtubes and stored at $-20{ }^{\circ} \mathrm{C}$. Tissues of a CVL-seronegative dog from a non-endemic area were used as negative controls, and in the analytical sensitivity experiments.

\section{Preparation of Leishmania and canine tissue suspensions}

Promastigotes of Leishmania spp. reference strains were concentrated and re-suspended in $100 \mu \mathrm{L}$ of Schneider medium and used for DNA extraction.

Aspirates of bone marrow and spleen fragments of the seronegative dogs were homogenized in TE $20 \% \mathrm{w} / \mathrm{v}(10 \mathrm{mM}$ Tris- $\mathrm{HCl} \mathrm{pH} 8.0 ; 1$ $\mathrm{mM}$ EDTA $\mathrm{pH}$ 8.0) and the suspensions were mixed with decreasing amounts of Leishmania (Leishmania) infantum chagasi promastigotes (MHOM/BR/2002/LPC-RPV) (see below).

A suspension containing $1.0 \times 10^{4}$ parasites/ $\mu \mathrm{L}$ of promastigotes of Leishmania (Leishmania) infantum chagasi (MHOM/BR/2002/ LPC-RPV) in Schneider medium was prepared (counted in a Neubauer chamber), then tenfold dilutions using TE buffer to a final volume of 20 $\mu \mathrm{L}$ were prepared. The dilution procedure was performed in duplicate.

Each serially diluted Leishmania suspension was mixed with either $100 \mu \mathrm{L}$ of bone marrow suspension or spleen suspension obtained from the seronegative dog, resulting in eight suspensions of bone marrow and eight suspensions of spleen containing from 200,000 to 0.02 promastigotes in a final volume of $120 \mu \mathrm{L}$.

\section{DNA extraction}

The bone marrow and spleen suspensions containing decreasing amounts of Leishmania promastigotes were washed twice in TE by centrifugation at $12,000 \mathrm{X} \mathrm{g}$ for $5 \mathrm{~min}$, the supernatant was discarded and the pellet was re-suspended in $500 \mu \mathrm{L}$ of lysis buffer $(10 \mathrm{mM}$ Tris$\mathrm{HCl}$ pH 8.0; 25 mM EDTA pH 8.0; 100 mM NaCl, $1 \%$ SDS, $10 \mu \mathrm{g} / \mathrm{mL}$ proteinase K). The suspension was incubated at $37{ }^{\circ} \mathrm{C}$ overnight. The DNA extraction was performed according to a phenol, chlorophorm, isoamyl-alcohol (25:24:1) protocol followed by a precipitation step with ethanol as described elsewhere ${ }^{14}$. The precipitated DNA was re-suspended in $30 \mu \mathrm{L}$ of TE and stored at $-20{ }^{\circ} \mathrm{C}$ until used in PCR.

Aspirates of bone marrow and spleen fragments of the 73 seropositive dogs were homogenized in TE $20 \% \mathrm{w} / \mathrm{v}(10 \mathrm{mM}$ Tris- $\mathrm{HCl} \mathrm{pH} 8.0 ; 1$ mM EDTA pH 8.0) and $100 \mu \mathrm{L}$ of the resulting suspensions were used in the DNA extraction, as previously described.

\section{Primers}

Five sets of primers were used: one set directed to the minicircleskDNA, two sets directed to the mitochondrial maxicircle-kDNA and two sets directed to the nuclear DNA. The primers for the minicircles-kDNA were described by Rodgers et al. (1990) $)^{15}$. The primers targeting the 


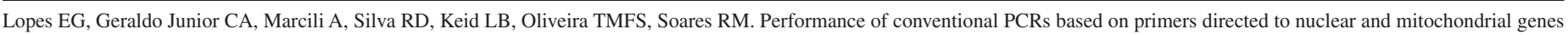
for the detection and identification of Leishmania spp. Rev Inst Med Trop Sao Paulo. 2016;58:41.

mitochondrial genes were based on cytochrome $\mathrm{B}(\mathrm{c} y t B)$ and cytochrome oxidase subunit II (coxII) gene sequences (maxicircle-kDNA). The primers directed to the nuclear DNA were based on the 18S rRNA sequence and cytochrome $\mathrm{C}(\mathrm{cyt} C)$ coding sequences. The primers targeting $c y t B$, coxII, and $c y \mathrm{tC}$ genes were designed in this study, and were based on sequences of several species of the genus Leishmania available in GenBank database. The sequences obtained from GenBank were aligned and consensus primers were designed to amplify gene fragments of all the sequences, indiscriminately. The primers directed to $18 \mathrm{~S}$ rRNA (for PCR and sequencing) were described elsewhere ${ }^{16}$. All the primers used in this study are described in Table 1.

\section{PCR}

The PCR cycling conditions were $94{ }^{\circ} \mathrm{C}$ for $3 \mathrm{~min}$, followed by 40 cycles of $94{ }^{\circ} \mathrm{C}$ for $30 \mathrm{~s}, \mathrm{~T}^{\circ} \mathrm{C}$ for $30 \mathrm{~s}$ and $72{ }^{\circ} \mathrm{C}$ for $30 \mathrm{~s}$ ( $\mathrm{T}$ value for each set of primers is found in Table 1). The PCR was ended with a final extension of $72{ }^{\circ} \mathrm{C}$ for $5 \mathrm{~min}$. Primers, $\mathrm{dNTP}$ and $\mathrm{MgCl}_{2}$ were used at a final concentration of $0.5 \mu \mathrm{M}, 200 \mu \mathrm{M}$ and $1.5 \mathrm{mM}$, respectively. Taq DNA polymerase platinum (Invitrogen ${ }^{\mathrm{TM}}$, Carlsbad, CA, USA) was used at a final concentration of 1.25 Units $/ 50 \mu \mathrm{L}$. Five microliters of the buffer supplied with the enzyme and $5 \mu \mathrm{L}$ of template DNA were added to the PCR mixture. To visualize the amplified products, $10 \mu \mathrm{L}$ of samples were mixed with $3 \mu \mathrm{L}$ of loading buffer and subject to electrophoresis in $2.0 \%$ agarose gels stained with ethidium bromide $(0.5 \mu \mathrm{g} / \mathrm{mL})$.

\section{Sequencing}

The PCR products were sequenced using the same primers and the Big Dye ${ }^{\circledR}$ reagent (Applied Biosystems, Foster City, CA, USA). Sequencing products were analyzed on an ABI377 automated sequencer. Both strands of each PCR product were sequenced at least four times in both directions to increase the reliability of sequencing. The sequences were assembled and the contig formed with the phred-base calling were analyzed using the phrap-assembly tool available in the suite Codoncode aligner v.1.5.2. (Codoncode Corp. Dedham, Massachusetts). The PCR derived sequences were submitted to the BLAST search (blastn, www. ncbi.nlm.nig.gov/BLAST) and similar sequences were downloaded to be used in the sequence analysis. Genetic sequences were deposited in GenBank under the accession numbers: KF302704 to KF302753; KU674349, KU674350.

\section{Sequence analysis}

The evolutionary history was inferred using the Neighbor-Joining method ${ }^{17}$. The percentage of replicate trees in which the associated taxa clustered together in the bootstrap test (1,000 replicates) were calculated as described elsewhere ${ }^{18}$. The tree was drawn to scale, with branch lengths in the same units as those of the evolutionary distances used to infer the phylogenetic tree. The evolutionary distances were computed using the Maximum Composite Likelihood method ${ }^{19}$. All positions containing gaps and missing data were eliminated from the dataset (complete deletion option). Phylogenetic analyses were conducted by using MEGA5 (maximum likelihood, evolutionary distance, and maximum parsimony methods $)^{20}$.

\section{Tests of samples}

Promastigotes of Leishmania spp. were tested with all the sets of primers, as well as the promastigote-spiked samples. The PCR products were sequenced, and these sequences were analyzed as already described. Aspirated bone marrow and spleen suspension from the 73 seropositive dogs were also tested by PCR using the minicircle-kDNA primers and the ones directed to cytochrome coding sequences.

\section{Ethics}

This work is in agreement with the Ethical Principles in Animal Research adopted by the Bioethics Commission of the School of Veterinary Medicine and Animal Science, University of Sao Paulo, Brazil (protocol number 1501/2008).

Table 1

Nucleotide sequences of the primers used in the molecular analysis, the fragment length they amplify, and annealing temperature for each pair used in PCR

\begin{tabular}{lclc}
\hline Name & Gene & Sequence & Fragment lenght $/$ T value \\
\hline $13 \mathrm{~A}$ & kDNA & GTG GGG GAG GGG CGT TCT & $116 / 56^{\circ} \mathrm{C}$ \\
$13 \mathrm{~B}$ & kDNA & ATT TTA CAC CAA CCC CCA GTT & \\
$609 \mathrm{~F}$ & $18 \mathrm{~S}$ & CAC CCG CGG TAA TTC CAG C & $900 / 55^{\circ} \mathrm{C}$ \\
$706 \mathrm{R}$ & $18 \mathrm{~S}$ & TTG AGG TTA CAG TCT CAG & Sequencing primers \\
$1156 \mathrm{~F}$ & $18 \mathrm{~S}$ & CGT ACT GGT GCG TCA GAG G & Sequencing primers \\
$1156 \mathrm{R}$ & $18 \mathrm{~S}$ & CCT CTG ACG CAC CAG TAC G & $377 / 52^{\circ} \mathrm{C}$ \\
CytB/R2 & Cytochrome B & GAA CTT CKA CAA TAH ACA AAT CAT AAT A \\
CytB/F1 & Cytochrome B & ATG CAT TTR TTT TGT TTA CAT TAT TTT A & \\
CytOxII/R2 & Cytochrome Oxidase II & GCA TAA ATC CAT GTA AAA CAC CAC A & $602 / 52^{\circ} \mathrm{C}$ \\
CytOxII/F1 & Cytochrome Oxidase II & TGG CTT TTA TWT TAT CAT TTT GAA TG & $230 / 60^{\circ} \mathrm{C}$ \\
CytC/F3 & Cytochrome C & GYG GYG AGA AGC TGT TCA AG & \\
CytC/R2 & Cytochrome C & CGA CAT CTT CGT GCC AGG CAT AA & \\
\hline
\end{tabular}


Lopes EG, Geraldo Junior CA, Marcili A, Silva RD, Keid LB, Oliveira TMFS, Soares RM. Performance of conventional PCRs based on primers directed to nuclear and mitochondrial genes for the detection and identification of Leishmania spp. Rev Inst Med Trop Sao Paulo. 2016;58:41.

\section{Statistical analysis}

The agreement between the diagnostic techniques was evaluated using the kappa test, with the following definitions: no agreement $(\mathrm{k}<0)$, slight agreement $(0<\mathrm{k}<0.2)$, fair agreement $(0.2<\mathrm{k}<0.4)$, moderate agreement $(0.4<\mathrm{k}<0.6)$, substantial agreement $(0.6<\mathrm{k}<0.8)$ and almost perfect agreement $(\mathrm{k}>0.8)^{21}$.

\section{RESULTS}

All the reference strains were successfully detected using primers targeting the minicircle-kDNA, maxicircle-kDNA ( $c y t B$ and $c o x I I)$ and the nuclear genes ( $c y t C$ and $18 S r R N A$ ). The evolutionary histories of standard isolates used in this study were reconstructed by the analysis of the nucleotide sequences obtained by each PCR. The topology of the trees revealed that all the markers could unequivocally differentiate the species (Fig. 1).

However, the PCRs based on primers directed to $c y t B, c y t C$ and coxII were about 10 times less sensitive than the primers directed to the minicircle-kDNA. The PCR targeting $c y t B, c y t C$ and $c o x I I$ were able to detect, theoretically, 20 to 200 Leishmania parasites diluted in $120 \mu \mathrm{l}$ of suspension, while the PCR directed to the minicircle-kDNA detected two to 20 parasites. The procedures to determine the analytical sensitivity were performed twice. The analytical sensitivity of the primers directed do $18 \mathrm{~S}$ rRNA was not determined.

The number of positive samples detected by the primers targeting $c y t B, c y t C$, and $c o x I I$ genes was significantly lower than the number of positive samples revealed by the PCR targeting the minicircle-kDNA. Eighteen dogs tested positive by PCR targeting the minicircle-kDNA while only two dogs were positive by PCR based on $c y t B, c y t C$, and $c o x I I$ primers. The two dogs that had positive PCR results in both bone marrow and spleen samples using all of the primers, and the corresponding sequencing results revealed that Leishmania (Leishmania) infantum chagasi was the cause of infections.

The performance of PCR using minicircle-kDNA primers for Leishmania detection in bone marrow and spleen samples of the 73 seropositive dogs resulted in a poor agreement according to the kappa coefficient test $(\mathrm{k}=0.4)$. Six animals had both samples positive (spleen and bone marrow), eight dogs had positive only the bone marrow samples positive, and four animals had only the spleen samples positive (Table 2).

\section{DISCUSSION}

This work aimed to evaluate the analytical and diagnostic performance of PCR assays based on primers targeting conserved genes for DNA barcoding within the mitochondrial and the nuclear genome of Leishmania spp.

Genes encoding proteins of the cytochrome family are valuable markers for molecular/phylogenetic studies, as they allow the reconstruction of the evolutionary histories in various classes of organisms, including the genus Leishmania ${ }^{21,22,23}$. For this reason, we designed new primers targeting the mitochondrial DNA, in particular $c y t B$, and $c o x I I$. The $c y t C$ gene encodes a mitochondrial product, but it
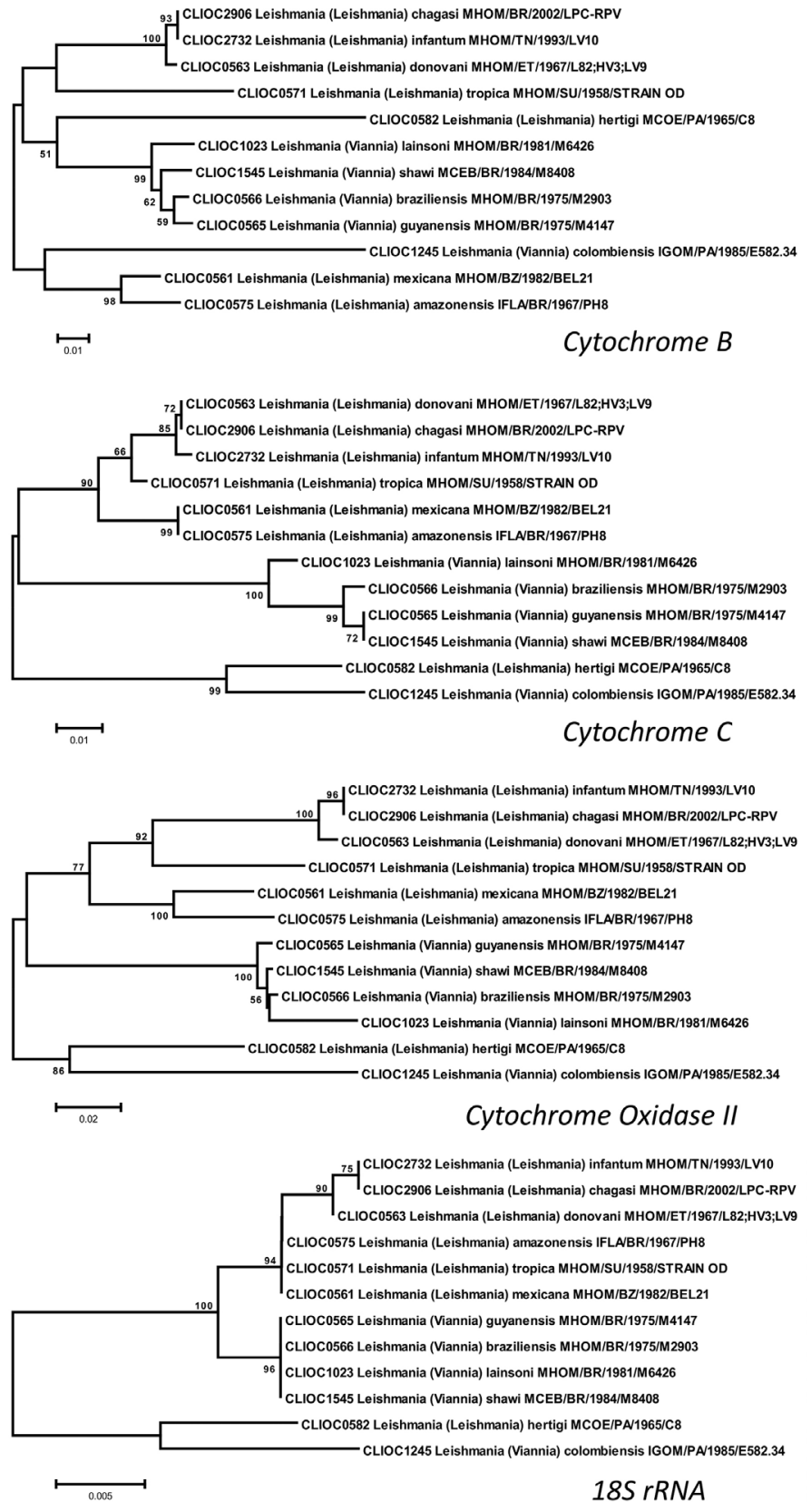

Fig. 1 - Evolutionary relationships within Leishmania spp. The evolutionary history was inferred using the Neighbor-Joining method. The percentage of replicate trees in which the associated taxa clustered together in the bootstrap test (1000 replicates) is shown next to the branches. The tree is drawn to scale, with branch lengths in the same units as those of the evolutionary distances used to infer the phylogenetic tree. The evolutionary distances were computed using the Maximum Composite Likelihood method and are in the units of the number of base substitutions per site. The analysis involved nucleotide sequences of locus coding for 18S rRNA, Citochrome B, Citochrome oxidase II and Citochrome C. All positions containing gaps and missing data were eliminated. Evolutionary analyses were conducted in MEGA5.

is located in the nuclear genome of the parasite ${ }^{25} . C y t C$ was selected for primer design because this gene also codes for a universally conserved protein and should have the same applicability in terms of phylogenetic studies as is the case of the genes located in the mitochondrial genome. 


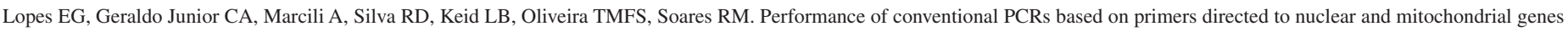
for the detection and identification of Leishmania spp. Rev Inst Med Trop Sao Paulo. 2016;58:41.

Table 2

Results of the PCR based on primers to minicircle-kDNA (PCR-kDNA) performed with samples of bone marrow and spleen from seropositive dogs for Canine Visceral Leishmaniasis

\begin{tabular}{lcccc}
\hline & & \multicolumn{2}{c}{ PCR-kDNA spleen } & Total \\
\cline { 2 - 5 } & & + & - & \\
\hline PCR-kDNA & + & 6 & 8 & 14 \\
bone marrow & - & 4 & 55 & 59 \\
\hline Total & & 10 & 63 & 73 \\
\hline
\end{tabular}

The experiment to determine the analytical specificity of PCRs targeting $c y t B, c y t C$ and $c o x I I$ showed that a broad range of species of the genus Leishmania could be successfully detected and identified. The topology of the trees reconstructed using each marker ( $c y t B, c y t C$, and coxII and $18 S r R N A$ ) were fully consistent when examined independently or compared two by two revealing that all the markers can be used together or separately for the molecular identification of Leishmania species.

The phylogeny of Leishmania isolates from China was reconstructed using coxII and cytB in two evolutionary studies ${ }^{26,27}$. CoxII phylogenies allowed the former authors to assign some of the autochthone isolates to define species within the genus Leishmania. In addition, a number of undescribed Leishmania species causing visceral leishmaniasis were identified by using $c y t B^{27}$. Phylogenies inferred with ITS-1, coxII and $c y t B$ had already been demonstrated as congruent ${ }^{26,27,28}$ and here we could extend these findings after adding more species to the analysis, and another marker, the $c y t C$. As pointed elsewhere ${ }^{26}$, the complementation of the molecular identification by using other mitochondrial and nuclear markers is advantageous for the microorganisms typing, and the four markers tested here have shown equal capability to discriminate each one of the well-defined species within the genus Leishmania. CytC seems to have the same evolutionary rate as $c y t B$, as judged from their equivalent overall similarity (not shown). Unlikely, coxII has shown a higher evolutionary rate, which has been reported elsewhere ${ }^{26}$.

Regarding the experiments on the analytical sensitivity of PCRs, a remarkable superiority of primers designed by Rodgers et al. (1990) $)^{15}$ was observed. The locus targeted by the primers $13 \mathrm{~A}$ and $13 \mathrm{~B}$ of the minicircle-kDNA is present in thousands of copies in the parasite genome, as well as the dozens of copies of the maxicircle-DNA hosting the $c y t B$ and $c o x I I$ genes $^{29}$. The higher number of copies could explain the higher analytical sensitivity of the PCR primers of the minicirclekDNA. Another important factor that could explain the differences in among the PCR assays is related to the molecular weight of the amplified products. It is well known that the PCR efficiency is greater when the amplified product is smaller ${ }^{30,31,32}$. The molecular weight of the amplified products with primers $13 \mathrm{~A}$ and $13 \mathrm{~B}$ are substantially smaller than the ones of the other PCRs.

Such a high difference in the PCR performances was also noted by Lachaud et al. (2002) ${ }^{33}$, when conventional PCR directed to kDNA had an absolute sensitivity 500-fold higher than the PCRs directed to the genes located in the nuclear genome (18S rRNA).

Considering the diagnostic sensitivity, the primers directed to the
minicircle-kDNA were more sensitive than the other primers. Based on these results, only two dogs were considered positive by the primers targeting the genes encoding cytochromes (cytB, $c y t C$ and $c o x I I)$.

The spleen and bone marrow kDNA-PCR results revealed that they were concordant in six of the 18 positive dogs, indicating that the use of only one type of sample for the detection of Leishmania spp. has a considerable chance of yielding a false negative result. Therefore, the association of two samples might be a necessary measure to increase the sensitivity of the molecular diagnosis of leishmaniasis.

Although molecular methods for CVL diagnosis based on nucleic acid amplification assays are considered highly sensitive ${ }^{34}$, in the present study the conventional PCR did not seem to be more sensitive than the serological techniques. In the present study, all the dogs tested positive by ELISA and IFAT (used consecutively), and this combination of tests was the official system used for the detection of infected dogs in Brazil until 2011. Nevertheless, seropositive results by ELISA and IFAT do not necessarily mean a true Leishmania spp. infection because of the controversial specificity of both tests. It is noteworthy that since 2011, the official criteria for the diagnosis of CVL in Brazil has been based on the use of a Dual Path Platform (DPP $®$ CVL, Biomanguinhos, Rio de Janeiro, RJ, Brazil) comprising specific recombinant proteins (rK26 and rK39) as the screening test, followed by the indirect ELISA as the confirmatory test ${ }^{35}$.

The determination of the sensitivity and specificity of diagnostic tests for CVL has been a moot point due to the difficulty in establishing a gold standard. The parasitological examination remains the gold standard for the diagnosis of CVL, and a positive result in any of the other diagnostic tests might be misinterpreted as a false positive, because of the low sensitivity of the parasitological exam.

The finding of a considerable amount of dogs that were positive by PCR performed on spleen samples, but negative in bone marrow samples and vice versa is a strong indication that PCR can fail quite often in detecting infected individuals. The characteristics of a diagnostic test for CVL may vary depending on the clinical condition of the $\operatorname{dog}^{36}$. In fact, the conventional PCR showed a low sensitivity for the detection of Leishmania spp. in samples of asymptomatic $\operatorname{dogs}^{34}$, and a strong association between the frequency of animals with symptoms and the frequency of positive animals by PCR has been shown ${ }^{37}$. Thus, although improving strategies for CVL diagnosis is important, samples should be carefully selected to avoid inappropriate interpretation of results.

In the present study, the detection thresholds of primers based on kDNA differed greatly from the results obtained by other authors. Nunes et al. $(2007)^{38}$ reported the ability to detect as few as 0.1 parasite in $500 \mu \mathrm{L}$ of spiked blood. In the present study, we were able to detect as few as two parasites in $120 \mu \mathrm{L}$ of bone marrow or spleen suspensions, corresponding to eight parasites in $500 \mu \mathrm{L}$. The differences in the two analytical sensitivities may be due to variances in the method of counting cells performed in both studies, as well as differences in the efficiency of DNA extraction, purification and nucleic acids concentration. Nevertheless, in both cases, it is evident that PCR does not seem to be a sensitive technique to detect infection in asymptomatic dogs. Whereas Nunes et al. $(2007)^{38}$ found 55 positive samples by PCR in a total of 80 blood samples from seropositive dogs (revealed by IFAT); we recorded 


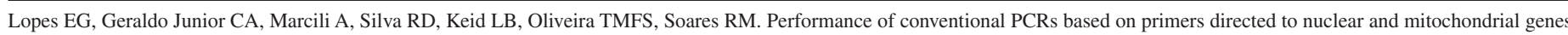
for the detection and identification of Leishmania spp. Rev Inst Med Trop Sao Paulo. 2016;58:41.

18 positive samples (in at least one of the tested samples) among the 73 seropositive dogs.

Lachaud et al. (2002) ${ }^{33}$ found a remarkable diagnostic sensitivity of a kDNA-PCR that was able to detect 25 seropositive, asymptomatic dogs among 29 tested animals. However, it is noteworthy that serology in this case was performed using two methods, IFAT and counter immune electrophoresis, which has certainly conferred a high positive predictive value to the serologic investigation.

There is no consensus among authors, particularly in Brazil, on which primers are to be used for the diagnosis of CVL as there are a multitude of oligonucleotide sequences available for the molecular diagnosis of this disease. Procedures for nucleic acids extraction have different performances, as well as methods for the handling of samples used in molecular diagnosis. Different performances of molecular techniques in several laboratories, make it difficult to compare results.

Carry-over contamination by amplicons is an issue that is rarely discussed by authors worldwide ${ }^{33}$ when they present results of surveys based on PCR and certainly has to be considered in the interpretation of techniques of exquisite sensitivity as is the case of amplification assays. The use of negative controls at various stages, disposable gloves frequently exchanged between the stages of the molecular procedures, automatic pipettes coupled to filter tips, and physical separation between working areas (DNA extraction, DNA amplification and manipulation of amplified products) are measures which are not always observed by technicians and, can seriously compromise the reliability of the results obtained by molecular techniques. In the present study, in addition to all the above measures, we used, in all stages of samples manipulation, at least one sample of ultrapure water for each three tested samples. The ultrapure water samples were used throughout the procedure.

Molecular diagnosis of CVL is considered successful if real time PCR is used ${ }^{34,39}$. In this case, the safety measures mentioned above should be even more rigorous, since the real-time PCR is usually performed in microtiter plates and many test samples are manipulated simultaneously, making the whole process more susceptible to cross contamination.

The results presented here allow us to conclude that the primers directed to $c y t B, c y t C$, and $\operatorname{coxII}$ can be used to identify isolates of parasites of the genus Leishmania, when used in combination with an automated sequencing technique. The conventional PCR is a technique that may have a low sensitivity for detection of infected dogs due to the possibility of generating a non-negligible number of false negative results when asymptomatic dogs infected by Leishmania are tested. Finally, primers based on the kDNA should be preferred for the screening of infected dogs.

\section{ACKNOWLEDGMENT}

R. M. Soares holds a productivity fellowship from CNPq.

\section{REFERENCES}

1. Lainson R, Shaw JJ. New World leishmaniasis. In Cox FEG, Wakelin D, Gillespie SH, Despommier DD, editors. Topley \& Wilson microbiology and microbial infections. London: Hodder Arnold; 2005. p. 313-49.
2. Santos-Gomes GM, Campino L, Abranches P. Canine experimental infection intradermal inoculation of Leishmania infantum promastigotes. Mem Inst Oswaldo Cruz. 2000;95:193-8.

3. Baneth G. Leishmaniasis. In Greene CE, editor. Clinical and infectious diseases of the dog and cat. Philadelphia: Elsevier; 2006. p. 685-98.

4. Degrave W, Fernandes O, Campbell D, Bozza M, Lopes U. Use of molecular probes and PCR for detection and typing of Leishmania: a mini-review. Mem Inst Oswaldo Cruz. 1994;89:463-9.

5. Ashford RW, Bates PA. Leishmaniasis in the Old World. In: Cox FEG, Kreier JP, Wakelin D, editors. Topley \& Wilson microbiology and microbial infections. New York: Wiley Blackwell; 1998. p. 215-40.

6. Galati EAB, Nunes VBL, Rego Jr, FA, Oshiro ET, Chang MR. Estudo de flebotomíneos (Diptera: Psychodidae) em foco de leishmaniose visceral no Estado do Mato Grosso do Sul, Brasil. Rev Saude Publica. 1997;31:378-90.

7. Lainson R, Rangel E. Lutzomyia longipalpis and the eco-epidemiology of American visceral leishmaniasis, with particular reference to Brazil: a review. Mem Inst Oswaldo Cruz. 2005;100:811-27.

8. Mauricio IL, Howard MK, Stothard JR, Miles MA. Genomic diversity in the Leishmania donovani complex. Parasitology. 1999;119(Pt 3):237-46.

9. Brasil. Ministério da Saúde. Secretaria de Vigilância Sanitária em Saúde. Departamento de Vigilância Epidemiológica. Manual de vigilância da leishmaniose tegumentar americana. Brasília: Ministério da Saúde; 2007.

10. Hebert PD, Ratnasingham S, deWaard JR. Barcoding animal life: cytochrome c oxidase subunit 1 divergences among closely related species. Proc Biol Sci. 2003;270 Suppl 1:S96-9.

11. Marcili A, Sperança MA, da Costa AP, Madeira M de F, Soares HS, Sanches C de $\mathrm{O}$, et al. Phylogenetic relationships of Leishmania species based on trypanosomatid barcode (SSU rDNA) and gGAPDH genes: taxonomic revision of Leishmania (L.) infantum chagasi in South America. Infect Genet Evol. 2014;25:44-51.

12. Escalante AA, Freeland DE, Collins WE, Lal AA. The evolution of primate malaria parasites based on the gene encoding cytochrome $\mathrm{b}$ from the linear mitochondrial genome. Proc Natl Acad Sci USA. 1998;95:8124-9.

13. Meyer A. Shortcomings of the cytochrome b gene as a molecular marker. Trends Ecol Evol. 1994;9:278-80.

14. Sambrook J, Fritsch EF, Maniatis T. Molecular cloning: a laboratory manual. New York: Cold Spring Harbor Laboratory Press; 1989.

15. Rodgers MR, Popper SJ, Wirth DF. Amplification of kinetoplast DNA as a tool in the detection disgnosis of Leishmania. Exp Parasitol. 1990;71:267-75.

16. Da Silva FM, Noyes H, Campaner M, Junqueira AC, Coura JR, Añez N, et al. Phylogeny, taxonomy and grouping of Trypanosoma rangeli isolates from man, triatomines and sylvatic mammals from widespread geographical origin based on SSU and ITS ribosomal sequences. Parasitology. 2004;129(Pt5):549-61.

17. Saitou N, Nei M. The neighbor-joining method: a new method for reconstructing phylogenetic trees. Mol Biol Evol. 1987;4:406-25.

18. Felsenstein J. Confidence limits on phylogenies: an approach using the bootstrap Evolution. 1985;39:783-91.

19. Tamura K, Nei M, Kumar S. Prospects for inferring very large phylogenies by using the neighbor-joining method. Proc Nat Acad Sci USA. 2004;101:11030-5.

20. Tamura K, Peterson D, Peterson N, Stecher G, Nei M, Kumar S. MEGA5: molecular evolutionary genetics analysis using maximum likelihood, evolutionary distance, and maximum parsimony methods. Mol Biol Evol. 2011;28:2731-9. 

for the detection and identification of Leishmania spp. Rev Inst Med Trop Sao Paulo. 2016;58:41.

21. Landis JR, Koch GG. The measurement of observer agreement for categorical data Biometrics. 1977;33:159-74.

22. Ibrahim ME, Barker DC. The origin and evolution of the Leishmania donovan complex as inferred from a mitochondrial cytochrome oxidase II gene sequence. Infect Genet Evol. 2001;1:61-8.

23. Luyo-Acero GE, Uezato H, Oshiro M, Takei K, Kariya K, Katakura K, et al. Sequence variation of the cytochrome $b$ gene of various human infecting members of the genus Leishmania and their phylogeny. Parasitology. 2004;128(Pt 5):483-91.

24. Asato Y, Oshiro M, Myint CK, Yamamoto Y, Kato H, Marco JD, et al. Phylogenic analysis of the genus Leishmania by cytochrome B gene sequencing. Exp Parasitol. 2009;121:352-61.

25. Rogers MB, Hilley JD, Dickens NJ, Wilkes J, Bates PA, Depledge DP, et al Chromosome and gene copy number variation allow major structural change between species and strains of Leishmania. Genome Res. 2011;21:2129-42.

26. Cao DP, Guo XG, Chen DL, Chen JP. Species delimitation and phylogenetic relationships of Chinese Leishmania isolates reexamined using kinetoplas cytochrome oxidase II gene sequences. Parasitol Res. 2011;109:163-73.

27. Yang BB, Chen DL, Chen JP, Liao L, Hu XS, Xu JN. Analysis of kinetoplast cytochrome $\mathrm{b}$ gene of 16 Leishmania isolates from different foci of China: different species of Leishmania in China and their phylogenetic inference. Parasit Vectors. $2013 ; 6: 32$.

28. Yang BB, Guo XG, Hu XS, Zhang JG, Liao L, Chen DL, et al. Species discrimination and phylogenetic inference of 17 Chinese Leishmania isolates based on internal transcribed spacer 1 (ITS1) sequences. Parasitol Res. 2010;107:1049-65.

29. Liu B, Liu Y, Motyka SA, Agbo EE, Englund PT. Fellowship of the rings: the replication of kinetoplast DNA. Trends Parasitol. 2005;21:363-9.

30. Garson JA, Ring CJ, Tuke PW. Improvement of HCV genome detection with "short" PCR products. Lancet. 1991;338(8780):1466-7.

31. Kleter B, van Doorn LJ, ter Schegget J, Schrauwen L, van Krimpen K, Burger M, et al. Novel short-fragment PCR assay for highly sensitive broad-spectrum detection of anogenital human papillomaviruses. Am J Pathol. 1998;153:1731-9.
32. Silva SO, Richtzenhain LJ, Barros IN, Gomes AM, Silva AV, Kozerski ND, et al. A new set of primers directed to $18 \mathrm{~S}$ rRNA gene for molecular identification of Cryptosporidium spp. and their performance in the detection and differentiation of oocysts shed by synanthropic rodents. Exp Parasitol. 2013;135:551-7.

33. Lachaud L, Chabbert E, Dubessay P, Dereure J, Lamothe J, Dedet JP, et al. Value of two PCR methods for the diagnosis of canine visceral leishmaniasis and the detection of asymptomatic carriers. Parasitology. 2002;125(Pt 3):197-207.

34. Ramos RAN, Ramos CAN, Jusi MMG, de Araújo FB, Machado RZ, Faustino MAG, et al. Polymerase chain reaction and real-time PCR for diagnosing of Leishmania infantum chagasi in dogs. Rev Bras Parasitol Vet. 2012;21:192-5.

35. Brasil. Ministério da Saúde. Secretaria de Vigilância em Saúde. Departamento de Vigilância das Doenças Transmissíveis. Esclarecimentos sobre substituição do protocolo diagnóstico da leishmaniose visceral canina (LVC). Brasília: Coordenação Geral de Doenças Transmissíveis/Coordenação Geral de Laboratórios de Saúde Pública; 2011. (Nota Técnica Conjunta nº 1 ).

36. Reis LE, Coura-Vital W, Roatt BM, Bouillet LÉ, Ker HG, Fortes de Brito RC, et al Molecular diagnosis of canine visceral leishmaniasis: a comparative study of three methods using skin and spleen from dogs with natural Leishmania infantum infection. Vet Parasitol. 2013;197:498-503.

37. Teles NM, Agostini MA, Bigeli JG, Noleto RV, Oliveira JD, de Oliveira Junior WP. Molecular and parasitological detection of Leishmania spp. in dogs caught in Palmas, TO, Brazil. Rev Bras Parasitol Vet. 2012;21:278-82.

38. Nunes CM, Dias AKK, Gottardi FP, De Paula HB, Azevedo MAA, Lima VM, et al Avaliação da reação em cadeia pela polimerase para o diagnóstico da leishmaniose visceral em sangue de cães. Rev Bras Parasitol Vet. 2007;16:5-9.

39. Francino O, Altet L, Sánchez-Robert E, Rodriguez A, Solano-Gallego L, Alberola $\mathrm{J}$, et al. Advantages of real-time PCR assay for diagnosis and monitoring of canine leishmaniosis. Vet Parasitol. 2006;137:214-21.

Received: 06 May 2015

Accepted: 26 November 2015 